\title{
Direct Coulomb and Exchange Interaction in Artificial Atoms
}

\author{
S. Tarucha, ${ }^{1,2, *}$ D. G. Austing, ${ }^{2}$ Y. Tokura, ${ }^{2}$ W. G. van der Wiel, ${ }^{3}$ and L. P. Kouwenhoven ${ }^{3, *}$ \\ ${ }^{1}$ Department of Physics, University of Tokyo, 7-3-1, Hongo, Bunkyo-ku, Tokyo 113-0033, Japan \\ ${ }^{2}$ NTT Basic Research Laboratories, 3-1, Morinosato Wakamiya, Atsugi-shi, Kanagawa 243-0198, Japan \\ ${ }^{3}$ Department of Applied Physics and DIMES, Delft University of Technology, P.O. Box 5046, 2600 GA Delft, The Netherlands
}

(Received 7 September 1999)

We determine contributions from the direct Coulomb and exchange interactions to the total interaction in artificial semiconductor atoms. We tune the relative strengths of the two interactions and measure them as a function of the number of confined electrons. The electrons tend to have parallel spins when they occupy nearly degenerate single-particle states. We use a magnetic field to adjust the single-particle-state degeneracy, and find that the spin configurations in an arbitrary magnetic field are well explained in terms of two-electron singlet and triplet states.

PACS numbers: 73.20.Dx, 72.20.My, 73.40.Gk

The addition of a single-electron charge to a quantum box costs a certain energy, which is responsible for Coulomb blockade in electron transport [1]. Also a change in spin is associated with a certain change in energy, e.g., exchange energy is gained when electrons are added with parallel spins as compared to antiparallel spins. Depending on the system, a large total spin (ferromagnetic filling) or a minimum total spin value (antiferromagnetic filling) is favored. In semiconductor quantum dots alternate spin filling [2] as well as spin-polarized filling [3] have been reported. Here. we study vertical quantum dots which have well-defined single-particle states. When these states are separated by a large energy, $\Delta E$, an antiferromagnetic filling is favored. For small $\Delta E$, ferromagnetic filling is observed, which is in line with Hund's first rule from atomic physics. We use a magnetic field $B$ to tune $\Delta E(B)$ allowing us to alter the spin filling.

We first discuss a simple model that describes filling of two single-particle states with two interacting electrons. Figure 1(b) shows two, spin-degenerate single-particle states with energies $E_{a}$ and $E_{b}$ crossing each other at $B=B_{0}$. The ground state (GS) energy $U(1)$, for one electron occupying these states, equals $E_{a}$ for $B<B_{0}$ and $E_{b}$ for $B>B_{0}$ [thick line in Fig. 1(b)]. For two electrons we can distinguish four possible configurations with either total spin $S=0$ (spin-singlet) or $S=1$ (spintriplet). (We neglect the Zeeman energy difference between $S_{z}=-1,0$, and 1.) The corresponding energies, $U_{i}(2, S)$ for $i=1$ to 4 , are given by $U_{1}(2,0)=$ $2 E_{a}+C_{a a}, \quad U_{2}(2,0)=2 E_{b}+C_{b b}, \quad U_{3}(2,1)=E_{a}+$ $E_{b}+C_{a b}-\left|K_{a b}\right|, \quad U_{4}(2,0)=E_{a}+E_{b}+C_{a b}+\left|K_{a b}\right|$. Here, $C_{i j}(i, j=a, b)$ is the direct Coulomb (DC) energy between two electrons occupying states with energies $E_{i}$ and $E_{j}$, and $K_{a b}$ is the exchange (EX) energy $\left(K_{a b}<0\right)$ between two electrons occupying $E_{a}$ and $E_{b}$ with parallel spins [4].

The experiments below measure the electrochemical potential defined for a two-electron system as $\mu(2) \equiv$ $U(2)-U(1)$. For each $U_{i}(2)$ we obtain the potentials: $\mu_{i}(2)=U_{i}(2)-E_{a} \quad$ for $\quad B<B_{0} \quad$ and $\quad \mu_{i}(2)=$
$U_{i}(2)-E_{b}$ for $B>B_{0}$ [see Fig. 1(c)]. The GS has $S=0$ away from $B_{0}$. Near $B_{0}$, the lowest energy is $\mu_{3}(2)$, such that $S=1$. The downward cusp in the thick line identifies this spin-triplet region. The transition in the GS from $S=0$ to 1 and $S=1$ to 0 , respectively, occurs when $\mu_{1}=\mu_{3}$ for $B<B_{0}$ and when $\mu_{2}=\mu_{3}$ for
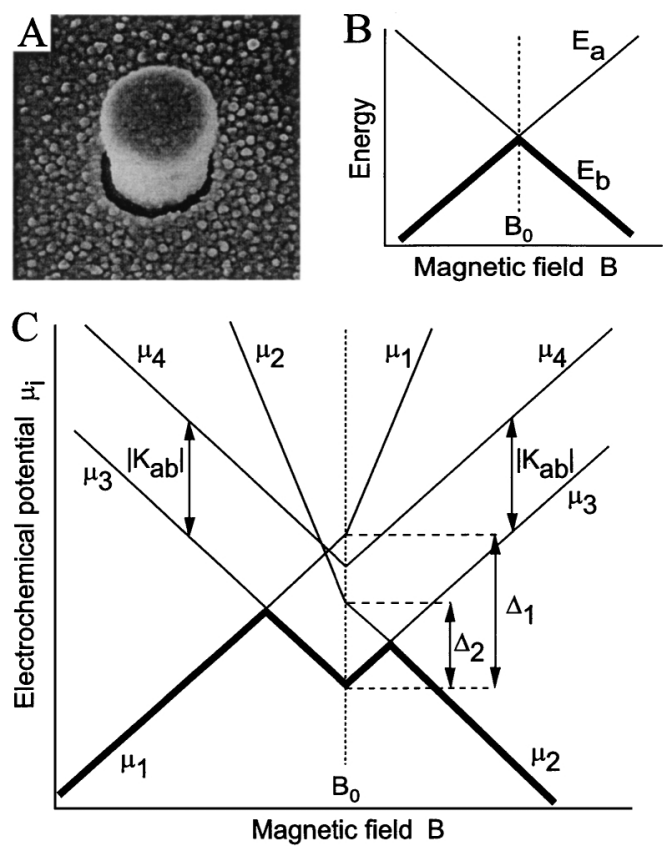

FIG. 1. (a) Scanning electron micrograph of the semiconductor quantum dot device. The quantum dot is located inside the $0.54-\mu \mathrm{m}$-diameter pillar and is made from a double-barrier structure [2]. The top and bottom contacts serve as source and drain electrodes. A Schottky gate is wrapped around the pillar. The current $I$ flowing through the dot is measured as a function of gate voltage $V_{g}$ in response to a dc voltage $V$ applied between the source and drain. (b) Schematic diagram of two single-particle states with energies $E_{a}$ and $E_{b}$ crossing each other at a magnetic field $B=B_{0}$. (c) Electrochemical potential, $\mu_{i}(2)=U_{i}(2)-U(1)$, for two interacting electrons. The thick line depicts the ground state energy, whereas the thin lines show the excited states. 
$B>B_{0}$. We define two energies, $\Delta_{1}$ and $\Delta_{2}$, to characterize the size of the downward cusp in the GS at $B=B_{0}$ : $\Delta_{1}=\mu_{1}-\mu_{3}=C_{a a}-C_{a b}+\left|K_{a b}\right|, \quad \Delta_{2}=\mu_{2}-\mu_{3}=$ $C_{b b}-C_{a b}+\left|K_{a b}\right|$, and $\Delta_{1}-\Delta_{2}=C_{a a}-C_{b b}$.

Our semiconductor quantum dot [see Fig. 1(a)] has the shape of a two-dimensional disk [5]. For detecting the GSs, we set the source-drain voltage $V$ to a small value. The GSs and excited states (ESs) are both measured when $V$ is set to a value sufficiently greater than the excitation energy [6]. For small $V$, a series of current peaks results from changing the number of electrons in the dot $N$ one by one [1]. The position of a current peak for the transition from $N-1$ to $N$ measures the GS electrochemical potential $\mu(N)$. The sample is cooled down to about $100 \mathrm{mK}$.

Figure 2(a) shows the evolution of current peaks with magnetic field for $N=7$ to 16 . Features associated with a parabolic confining potential are all observed such as a shell structure [2]. The large spacing for $N=12$ at $B=0 \mathrm{~T}$ can be seen in Fig. 2(a) (see double arrow) and marks the complete filling of the first three shells. The pairing between neighboring peaks indicates antiparallel spin filling of a single orbital state by two electrons. Modifications to this pairing are observed for the peaks labeled by "ם" at $0 \mathrm{~T}$, and in each of the dashed ovals connecting pairs of peaks at nonzero field. These are all signatures of Hund's first rule; i.e., spin-polarized filling. Note
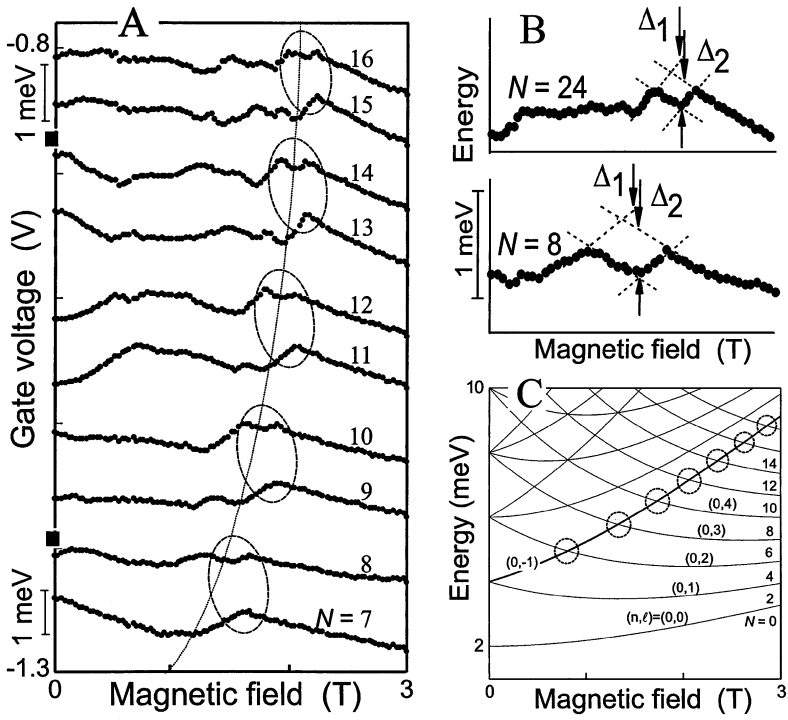

FIG. 2. (a) Evolution of the ground state energies from $N=7$ to 16 as measured from the current peaks versus magnetic field at $V=120 \mu \mathrm{V}$. The bars along the gate voltage axis show $1 \mathrm{meV}$ energy scales calibrated at -1.26 and $-0.85 \mathrm{~V}$. The dotted curve indicates the last crossing between single-particle states. Dashed ovals correlate pairs of ground states for odd and even electron numbers. Spin transitions in the ground states are indicated by " $\square$ " at $B=0 \mathrm{~T}$ and occur in the ovals for $B \neq$ 0 T. (b) Magnified plots of the $N=8$ and 24 current peaks vs magnetic field. The dashed lines illustrate how the interactionenergy parameters $\Delta_{1}$ and $\Delta_{2}$ are determined. (c) Fock-Darwin single-particle states calculated for $\hbar \omega_{0}=2 \mathrm{meV}$. that the Zeeman effect is negligible in this experiment [7]. We show expansions of the evolution of the $N=8$ and $N=24$ peaks in Fig. 2(b). The downward cusps are clearly seen. The dashed lines form a parallelogram, from which we obtain parameters $\Delta_{1}$ and $\Delta_{2}$.

To compare the two-electron model with larger electron numbers, we assume that other states are far away in energy so that they can be neglected. Then, the downward cusps should occur for higher even-electron numbers, whereas they should be absent for odd-electron numbers. This is clearly observed in the ovals in Fig. 2(a). For instance, the $B$-field dependence of the 9th peak compares well to the thick line in Fig. 1(b) and the $B$-field dependence of the 10th peak compares well to the thick line in Fig. 1(c). Other pairs of even and odd numbered peaks show the same behavior. This justifies our assumption so that we can simplify the many-electron system to just one or two electrons.

More detailed agreement is obtained by measuring the excitation spectrum [6]. Figure 3 shows a $d I / d V_{g}$ plot, taken for $V=2 \mathrm{mV}$. This larger voltage opens a sufficiently wide transport window between the Fermi levels of the source and drain, that both the GS and first few ESs can be detected. The GS and ESs for $N=7$ to 9 can be assigned from the magnetic field dependence of the dark blue lines. Solid red lines highlight the GSs, whereas the ESs are indicated by dashed red lines. The set of GS and ES lines for $N=7$ shows a single crossing similar to that in Fig. 1(b). The spectrum for $N=8$ compares well to

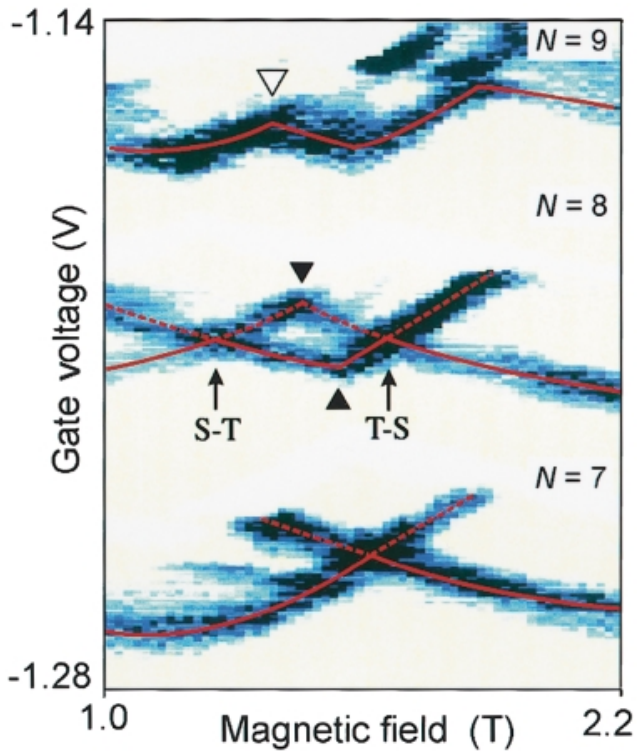

FIG. 3 (color). $d I / d V_{g}$ in the plane of $V_{g}$ and $B$ for $N=7$ to 9 measured for $V=2 \mathrm{mV}$. $d I / d V_{g}>0$ for dark blue, $d I / d V_{g}<0$ for white, and $d I / d V_{g} \approx 0$ for yellow. The solid red lines indicate the evolution of the GSs with magnetic field, whereas the dashed red lines show the ESs. The two arrows indicate singlet-triplet $(\mathrm{S}-\mathrm{T})$ and triplet-singlet $(\mathrm{T}-\mathrm{S})$ transitions in the GS for $N=8$ 
Fig. 1(c) and we can clearly distinguish the parallelogram formed by the GS and first ES. The downward cusp in the GS for $N=8$ (labeled " $\boldsymbol{\Delta}$ ") is at a slightly higher $B$ field than the upward cusp in the first ES (labeled " $\nabla$ "). This asymmetry implies that $\Delta_{1}>\Delta_{2}$, i.e., $C_{a a}>C_{b b}$. The same type of asymmetry is always observed along the dashed line in Fig. 2(a), implying that $C_{a a}>C_{b b}$ for all $N$. Note that the GS for $N=9$ shows an upward cusp (labeled by $\nabla$ ) quite similar in form to the first ES in the spectrum for $N=8$. This implies that the filling of the ninth electron is closely linked to the configuration of the $N=8$ first ES [3].

As illustrated in Fig. 2(b), we can derive the experimental values for $\Delta_{1}$ and $\Delta_{2}$ for different $N$. This values are plotted in Fig. 4. We find that $\Delta_{1}$ is larger than $\Delta_{2}$ for all $N$, again implying that $C_{a a}>C_{b b}$. As $N$ increases from 6 to $12, \Delta_{1}$ first increases and then slowly decreases while $\Delta_{2}$ slightly decreases.

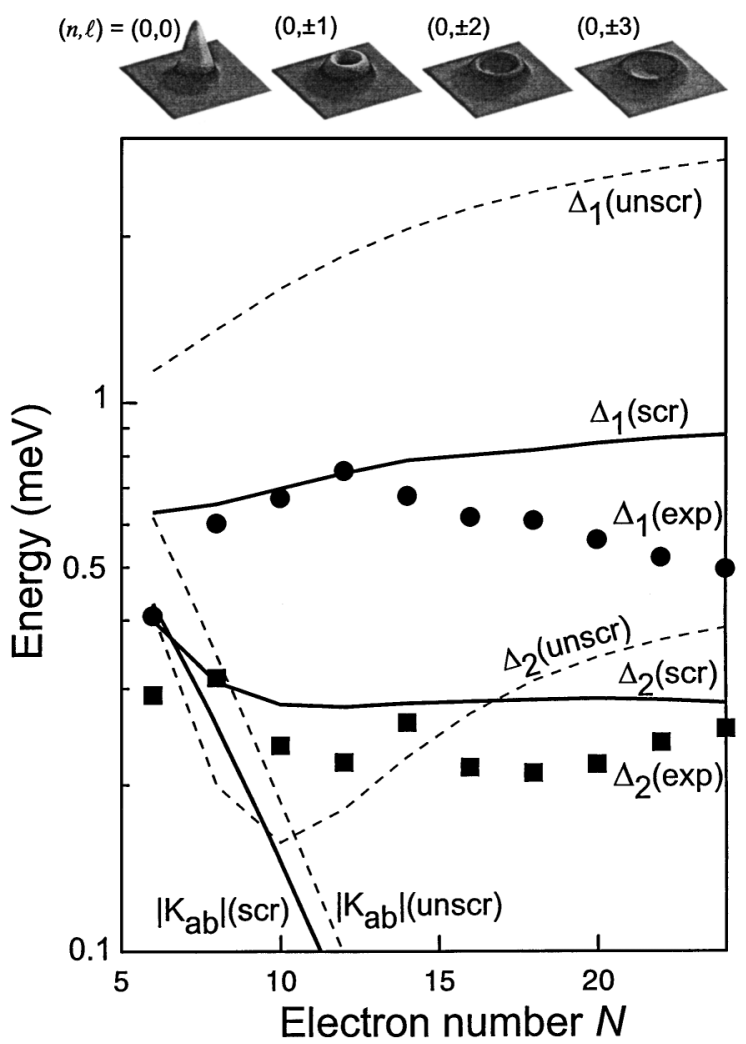

FIG. 4. Experimental values for the energy parameters $\Delta_{1}($ and $\Delta_{2}(\boldsymbol{\square})$, on a log scale, versus the electron number derived from data as shown in Fig. 2(a). The uncertainty in the determination of the experimental values is $\pm 10 \%$ or less. The dashed and solid curves are calculated from the FD wave functions at $2 \mathrm{~T}$, for an unscreened (unscr) and screened (scr) Coulomb interaction. The calculated exchange energy $\left|K_{a b}\right|$ between states with energies $E_{a}=E_{0,-1}$ and $E_{b}=E_{0, N / 2-1}$ decreases quickly with $N$. Above the main figure the absolute squares of the wave functions are shown for the relevant quantum numbers $n=0$ and $\ell=0, \pm 1, \pm 2, \pm 3$. As the angular momentum quantum number $\ell$ increases, the average radius increases.
To calculate DC and EX energies [4] we now need to specify the confining potential of the disk. In earlier work it was shown that the lateral confinement is well described by a parabolic potential with cylindrical symmetry [2]. The eigenfunctions with eigenenergies, $E_{n, l}$, in this potential are known as Fock-Darwin (FD) states [8]:

$$
E_{n, l}=-\frac{l}{2} \hbar \omega_{c}+\left(n+\frac{1}{2}+\frac{1}{2}|l|\right) \hbar \sqrt{4 \omega_{0}^{2}+\omega_{c}^{2}},
$$

where $n=0,1,2, \ldots$ is the radial quantum number and $l=0, \pm 1, \pm 2, \ldots$ is the quantum number for angular momentum. $\hbar \omega_{0}$ is the lateral confining energy and $\hbar \omega_{c}=e B / m^{*}$ is the cyclotron energy. Each FD state is spin degenerate. At $B=0 \mathrm{~T}$ the FD spectrum has sets of states with increasing degeneracy [see Fig. 2(c)]. This degeneracy is lifted on increasing $B$, but as $B$ is increased further, new crossings can occur. The last crossing is always a crossing between just two FD states. The up-going state is always $(n, l)=(0,-1)$, whereas the down-going state $(0, l>1)$ has an increasing angular momentum for states with increasing energy. (The relation with Fig. 1 is $E_{a}=E_{0,-1}$ and $E_{b}=E_{0, l>1}$.) Note that the last crossings also correspond to the dashed line in Fig. 2(a).

From the electron distributions of the FD states we calculate the DC and EX energies for two electrons occupying two degenerate states. We take $\hbar \omega_{0}=2 \mathrm{meV}$ as deduced from earlier experiments [2,6] and obtain $\Delta_{1}$ and $\Delta_{2}$. The dashed curves in Fig. 4 show $\Delta_{1}$ and $\Delta_{2}$ when we neglect screening of the interactions within the dot by electrons in the leads and in the gate. In this case the Coulomb potential falls off as $1 / r$, where $r$ is the distance between the electrons [4]. For the solid curves we have approximated the screening effects by replacing the Coulomb potential by $\exp (-r / d) / r$. We have taken $d=10 \mathrm{~nm}$ which is roughly the thickness of the tunnel barriers. Figure 4 shows that screening considerably reduces $\Delta_{1}$ to values much closer to the experimental values. Screening also removes the minimum in $\Delta_{2}$, which is also in better agreement with the experiment. Since the average radius of the wave functions increases with angular momentum, two electrons are closer together when they both occupy $(0,-1)$ compared to when they both occupy $(0, l=N / 2-1)$ for even $N>4$ (or $l>1$ ), so the DC interaction is stronger in the former. This explains our observation $C_{a a}>C_{b b}$ for all $N$. The overlap between different wave functions $(0,-1)$ and $(0, l=N / 2-1)$ decreases for even $N>4$ (or $l>1$ ). This results in a decrease in both $C_{a b}$ and $\left|K_{a b}\right|$ with $N$. It then follows that $\Delta_{1}$ increases until it saturates at a value equal to $C_{a a}$. The gradual decrease of experimental $\Delta_{1}$ for $N>12$ is probably related to the decrease in the lateral confinement with $N$ [2], and thus the decrease in $C_{a a}$.

We finally discuss the interaction effects for the $N=4$, 8 , and 14 peaks near $B=0 \mathrm{~T}$ [the $N=8$ and 14 peaks are labeled " $\square$ " in Fig. 2(a)]. These correspond to the 


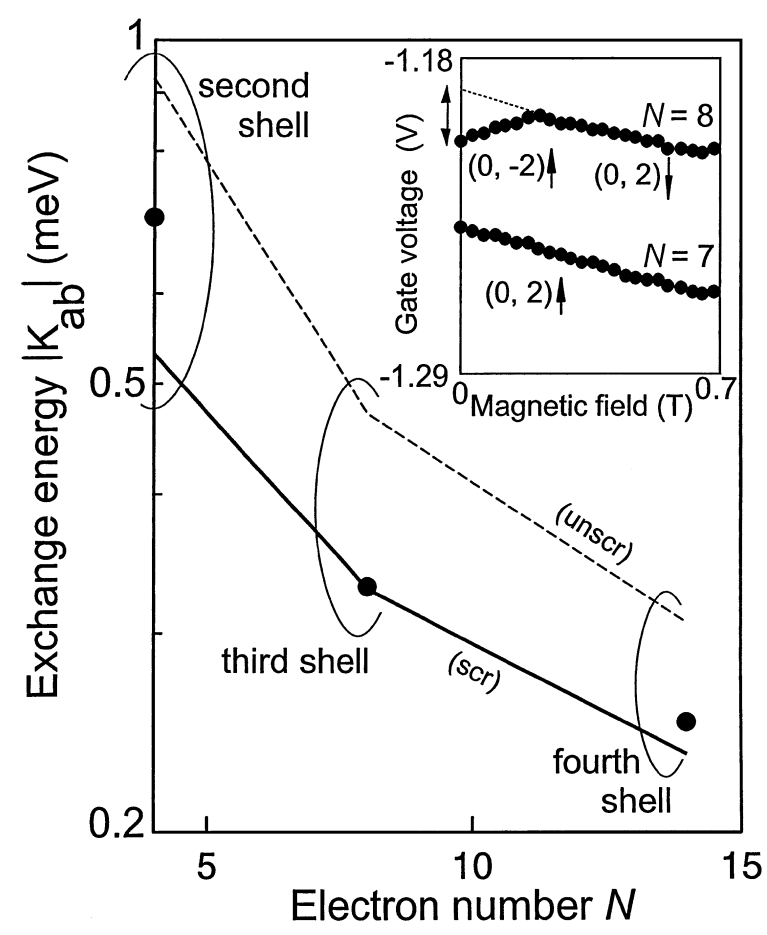

FIG. 5. Exchange energy $\left|K_{a b}\right|$, on a log scale, associated with spin triplets formed when each new shell is filled by just two electrons at $B=0 \mathrm{~T}$. The solid circles are the experimental values whose uncertainty in the determination is $\pm 10 \%$ or less. The inset shows an expansion for the filling of the first two electrons into the third shell (i.e., $N=7$ and 8 ). The vertical double arrow represents $\left|K_{a b}\right|$ in units of gate voltage which is then converted to energy. The calculated curves in the main figure are for the unscreened (dashed) and screened (solid) cases.

GS electrochemical potentials for adding the second electron to the second, third, and fourth shells, respectively. The inset to Fig. 5 demonstrates the resemblance to the model of Fig. 1(c) for $N=8$ near $B=0 \mathrm{~T}$. Comparing these data to the FD spectrum, we assign the states such that $E_{a}=E_{0,-2}$ and $E_{b}=E_{0,2}$. Likewise, for $N=4$ we have $E_{a}=E_{0,-1}$ and $E_{b}=E_{0,1}$ [2] and for $N=14$ we have $E_{a}=E_{0,-3}$ and $E_{b}=E_{0,3}$ [9]. Note that these states correspond to wave functions with a complete overlap. Also, for $B=B_{0}=0 \mathrm{~T}$ the two crossing states have the same orbital symmetry implying $\Delta_{1}=\Delta_{2}=\left|K_{a b}\right|$; i.e., only EX effects contribute to the downward cusp [10].

We derive $\left|K_{a b}\right|$ as illustrated in the inset to Fig. 5. The obtained EX energy quickly becomes smaller for higher lying shells. For comparison we also show the calculated screened and unscreened values. The screened case provides the best quantitative agreement for our realistic choices of the confining energy and the screening distance.

Our general model provides a clear identification of effects due to EX and DC interactions. More advanced calculations support our analyses [11]. An important simplification is the reduction of a many-electron system to just two interacting electrons. The type of spin filling in many nearly degenerate levels near $B=0 \mathrm{~T}$ in larger electron boxes remains an interesting open issue.

We thank G. Bauer, M. Danoesastro, M. Eto, R. van der Hage, T. Honda, J. Janssen, T. Oosterkamp, H. Tamura, and T. Uesugi for their help and useful discussions. S. T. and L.P.K. acknowledge financial support from the Specially Promoted Research, Grant-in-Aid for Scientific Research, from the Ministry of Education, Science and Culture in Japan, from the Dutch Organization for FOM, from the NEDO program NTDP-98, and from the EU via a TMR network.

*Also at ERATO Mesoscopic Correlation Project.

[1] L. P. Kouwenhoven et al., in Mesoscopic Electron Transport, edited by L. L. Sohn et al. (Kluwer, Dordrecht, The Netherlands, 1997), Ser. E, Vol. 345, p. 105.

[2] S. Tarucha et al., Phys. Rev. Lett. 77, 3631 (1996); S. Tarucha et al., Physica (Amsterdam) 3E, 112 (1998).

[3] D. R. Stewart et al., Science 278, 1784 (1997).

[4] $C_{i j}$ and $K_{i j}$ are the usual wave function overlap integrals for an unscreened Coulomb potential:

$$
\begin{aligned}
C_{i j}=\iint\left|\psi_{i}\left(\vec{r}_{1}\right)\right|^{2} \frac{e^{2}}{4 \pi \varepsilon_{0}\left|\vec{r}_{1}-\vec{r}_{2}\right|}\left|\psi_{j}\left(\vec{r}_{2}\right)\right|^{2} d \vec{r}_{1} d \vec{r}_{2}, \\
K_{i j}=-\iint \psi_{i}^{*}\left(\vec{r}_{1}\right) \psi_{j}\left(\vec{r}_{1}\right) \\
\quad \times \frac{e^{2}}{4 \pi \varepsilon_{0}\left|\vec{r}_{1}-\vec{r}_{2}\right|} \psi_{j}^{*}\left(\vec{r}_{2}\right) \psi_{i}\left(\vec{r}_{2}\right) d \vec{r}_{1} d \vec{r}_{2},
\end{aligned}
$$

$E_{i}$ and $E_{j}$ are eigenvalues for the eigenfunctions $\psi_{i}$ and $\psi_{j}$, respectively, and $\vec{r}_{1}$ and $\vec{r}_{2}$ represent the positions of the two electrons.

[5] D. G. Austing et al., Semicond. Sci. Technol. 11, 388 (1996).

[6] L. P. Kouwenhoven et al., Science 278, 1788 (1997).

[7] S. Sasaki et al., Physica (Amsterdam) 256B, 157 (1998).

[8] V. Fock, Z. Phys. 47, 446 (1928); C. G. Darwin, Proc. Cambridge Philos. Soc. 27, 86 (1930).

[9] For $N=14$ it is difficult to identify the corresponding state since the $B$ dependence is not clear enough to distinguish between $E_{0,-3}$ and $E_{1,-1}$. We assume it is $E_{0,-3}$, and continue to evaluate $\left|K_{a b}\right|$.

[10] Note that the asymmetry $\left(\Delta_{1} \neq \Delta_{2}\right)$ can occur only for $B_{0} \neq 0$. Symmetry is required if $B_{0}=0$, implying that $\Delta_{1}=\Delta_{2}$. Then, the eigenfunctions for the states with energies $E_{a}$ and $E_{b}$ must have the same spatial form.

[11] There are various calculations consistent with our experiment: The exact calculation for $N<7$ by A. Wojs and P. Hawrylak, Phys. Rev. B 53, 10841 (1996) and M. Eto, Jpn. J. Appl. Phys. 36, 3924 (1997); Hartree-Fock calculations for $N>8$ by A. Natori et al., Jpn. J. Appl. Phys. 36, 3960 (1997); H. Tamura, Physica (Amsterdam) 249B-251B, 210 (1998); and M. Rontani et al., Phys. Rev. B 59, 10165 (1999); the spin density-functional theory at $B=0 \mathrm{~T}$ by In-Ho Lee et al., Phys. Rev. B 57, 9035 (1998) and M. Koskinen, M. Manninen, and S. M. Reimann, Phys. Rev. Lett. 79, 1817 (1997); and also at nonzero $B$ by O. Steffen, U. Rossler, and M. Suhrke, Europhys. Lett. 42, 529 (1998). 